TRANSACTIONS OF THE

AMERICAN MATHEMATICAL SOCIETY

Volume 353, Number 11, Pages 4461-4479

S 0002-9947(01)02781-7

Article electronically published on May 3, 2001

\title{
EMBEDDINGS OF $\mathrm{DI}_{2}$ IN $\mathrm{F}_{4}$
}

\author{
CARLES BROTO AND JESPER M. MØLLER
}

\begin{abstract}
We show that there is only one embedding of $\mathrm{BDI}_{2}$ in $\mathrm{BF}_{4}$ at the prime $p=3$, up to self-maps of $\mathrm{BDI}_{2}$. We also describe the effect of the group of self-equivalences of $\mathrm{BF}_{4}$ at the prime $p=3$ on this embedding and then show that the Friedlander exceptional isogeny composed with a suitable Adams map is an involution of $\mathrm{BF}_{4}$ whose homotopy fixed point set coincide with $\mathrm{BDI}_{2}$
\end{abstract}

\section{INTRODUCTION}

A $p$-compact group is a $p$-complete analogue in homotopy theory of the concept of compact Lie group. The notion was introduced by Dwyer and Wilkerson in [13. A $p$-compact group is a $p$-complete space $B X$ whose loop space is $\mathbf{F}_{p}$-finite, i.e. has finite $\bmod p$ cohomology. The $p$-completion of the classifying space of a compact connected Lie group is an example of a $p$-compact group but there are other, exotic, examples. Among other notions that $p$-compact groups share with compact Lie groups are that of a maximal torus, Weyl group, and maximal torus normalizer (see [13] for the definitions). There is also a well defined concept of homomorphism or monomorphism between $p$-compact groups. Since the introduction of this concept one main issue has been the description of the exotic examples. These are simply connected and irreducible $p$-compact groups that do not appear as the $p$-completion of a compact Lie group. Contrary to what happens with compact Lie groups these exotic examples can only be defined as $p$-complete spaces at a certain given prime $p$. We will focus our attention on one of these examples, namely, $\mathrm{BDI}_{2}$. This is a $3-$ compact group with mod 3 cohomology ring

$$
H^{*}\left(\mathrm{BDI}_{2} ; \mathbf{F}_{3}\right) \cong P\left[x_{12}, x_{16}\right], \quad P^{1} x_{12}=x_{16},
$$

the rank two Dickson algebra at the prime 3. All of our calculations and results will be 3-primary. In particular we are considering the 3-completion of all spaces involved, thus we will omit explicit notation for this fact.

Theorem 1.1. Any non-trivial homomorphism from $\mathrm{DI}_{2}$ to $\mathrm{F}_{4}$ is a monomorphism and the group of automorphisms of $\mathrm{DI}_{2}$ acts simply transitively on the non-empty set of monomorphisms from $\mathrm{DI}_{2}$ to $\mathrm{F}_{4}$.

There is then a monomorphism $\mathrm{B} \alpha: \mathrm{BDI}_{2} \rightarrow \mathrm{BF}_{4}$ and it is unique up to composition with automorphisms of $\mathrm{DI}_{2}$. We can rephrase it by saying that $\mathrm{F}_{4}$ contains a unique copy of $\mathrm{DI}_{2}$.

Received by the editors May 4, 1999.

1991 Mathematics Subject Classification. Primary 55R35, 55P15, 55P10.

Key words and phrases. Homotopy Lie groups, Dickson algebra.

C. Broto is partially supported by DGES grant 97-0203. 
If we compose the Friedlander exceptional isogeny of $\mathrm{F}_{4}, \mathrm{~B} \varphi: \mathrm{BF}_{4} \rightarrow \mathrm{BF}_{4}$ [15], with the Adams map $\psi^{1 / \zeta}$, where $\zeta^{2}=-2$, then $\mathrm{B} \tau=\mathrm{B} \psi^{1 / \zeta} \circ \mathrm{B} \phi$ is a homotopy involution of $\mathrm{BF}_{4}$ that fixes $\mathrm{B} \alpha: \mathrm{BDI}_{2} \rightarrow \mathrm{BF}_{4}$ up to homotopy.

Theorem 1.2. There is an action of $\mathbf{Z} / 2$ on $\mathrm{BF}_{4}$ induced up to homotopy by $\mathrm{B} \tau$, for which $\mathrm{B} \alpha: \mathrm{BDI}_{2} \rightarrow \mathrm{BF}_{4}$ induces a homotopy equivalence

$$
\mathrm{BDI}_{2} \simeq\left(\mathrm{BF}_{4}\right)^{h \mathbf{Z} / 2}
$$

that identifies $\mathrm{BDI}_{2}$ with a homotopy fixed point space of $\mathrm{BF}_{4}$.

Notice that in particular the Bousfield-Kan spectral sequence for the homotopy groups of $\left(\mathrm{BF}_{4}\right)^{h \mathbf{Z} / 2}$ degenerates to

$$
\pi_{*}\left(\mathrm{BDI}_{2}\right) \cong \pi_{*}\left(\mathrm{BF}_{4}\right)^{\mathbf{Z} / 2}
$$

As a further corollary we obtain

Corollary 1.3. For any space $X, \operatorname{map}\left(X, \mathrm{BDI}_{2}\right) \simeq \operatorname{map}\left(X, \mathrm{BF}_{4}\right)^{h \mathbf{Z} / 2}$.

And applying this result to the homogeneous space $\mathrm{F}_{4} / \mathrm{DI}_{2}$,

Corollary 1.4 (Harper [16]). At the prime 3 there is a splitting

$$
\mathrm{F}_{4} \simeq \mathrm{DI}_{2} \times \mathrm{F}_{4} / \mathrm{DI}_{2} \text {. }
$$

Some historical considerations about the construction of this space will clarify our motivation. The way exotic examples are obtained is as realizations of graded polynomial algebras over $\mathbf{F}_{p}$ as mod $p$ cohomology rings of $p$-completed spaces $B X$ which will turn out to be $p$-compact groups. Steenrod [32] already pointed out the importance of realizing polynomial algebras as cohomology rings. The idea was reconducted by Clark-Ewing [9, Wilkerson [34, 36], and Adams-Wilkerson [2] who noticed the relevance of invariant theory to the realization problem.

Dickson algebras at odd primes are invariant algebras $D(n) \cong \mathbf{F}_{p}\left[t_{1}, \ldots, t_{n}\right]^{\mathrm{GL}_{n}\left(\mathbf{F}_{p}\right)}$ by the action of the full general linear group of $n$ by $n$ matrices over $\mathbf{F}_{p}$ on a polynomial algebra on $n$ independent generators of degree 2. They are shown to be again polynomial algebras of $n$ independent generators of degrees $2\left(p^{n}-p^{i}\right)$ [11, 29, 35]. In [29] the authors already proved that Dickson algebras at odd primes can only appear as a cohomology ring if $n=1$, in which case we obtain the cohomology of a $p$-local sphere of dimension $2(p-1)$ or in case $n=2$ and $p=3$,

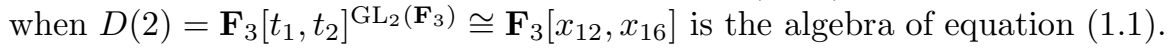

The existence and properties of DI(2) have attracted the attention of experts in the subject since the seventies. Much published and unpublished work has been done. We will only sketch some main achievements that can be found in the literature.

A space $\mathrm{BDI}_{2}$ realizing $D(2)$ as a mod 3 cohomology ring was first obtained by Zabrodsky in [38. His method was technical and quite involved. It consisted in realizing geometrically the 3-local homotopy classes of $\mathrm{BF}_{4}$, the classifying space of the exceptional Lie group $\mathrm{F}_{4}$, that remain invariant under the action of the Friedlander exceptional isogeny. A more intelligible construction was provided by Aguadé [3]. The structure of $\mathrm{BDI}_{2}$ as a 3-compact group was exposed in [23]. However one important feature of Zabrodsky's construction remained unexplained in these new approaches. Zabrodsky's construction came together with a map $\mathrm{BDI}_{2} \rightarrow \mathrm{BF}_{4}$, after 3-completion, turning $H^{*}\left(\mathrm{BDI}_{2} ; \mathbf{F}_{3}\right)$ into a finitely generated $H^{*}\left(\mathrm{BF}_{4} ; \mathbf{F}_{3}\right)$-module, thus representing a monomorphism of 3 -compact groups. The 
original motivation of this note was an attempt to explain not only the methods of Zabrodsky but the fact that $\mathrm{BDI}_{2}$ was obtained as a sort of invariant gadget in $\mathrm{BF}_{4}$. This is achieved in Theorem 1.1 and equation (1.2).

The paper is organized as follows. Sections 2 and 3 are devoted to a careful investigation of $p$-compact group homomorphisms from $\mathrm{DI}_{2}$ to $\mathrm{F}_{4}$. This leads to the proof of Theorem 1.1 In Section 4 we compute the cohomological structure of the homogeneous space $\mathrm{F}_{4} / \mathrm{DI}_{2}$. It turns out to coincide with that of Harper's molecule at the prime 3, and this, in turn implies that both spaces have the same homotopy type. Details are included in Section 7 for convenience of the reader. Sections 5 and 6 are devoted to the Friedlander exceptional isogeny of $\left(\mathrm{BF}_{4}\right)_{1 / 2}$. It is shown that suitably composed with an Adams map it becomes an involution that fixes $\mathrm{DI}_{2}$. The effect of these maps in cohomological generators is determined and this leads to the proof of Theorem 1.2 and Corollaries 1.3 and 1.4 Some straightforward calculations, mainly in Section 2, were done with the aid of a computer using the magma system. It should be easy to perform the same calculations with any other suitable program.

The authors are grateful to Bill Dwyer for a helpful discussion that led to the final form of Theorem[1.2, and also to Kasper Andersen for his help with the magma system. We kindly acknowledge the support of the Centre de Recerca Matemàtica of the Institut d'Estudis Catalans and the Matematisk Institut of the Københavns Universitet that made it possible for the authors to meet.

\section{Admissible HOMOMORPHISMS}

Let $X_{1}$ and $X_{2}$ be $p$-compact groups [13] with maximal tori $i_{1}: T_{1}=T\left(X_{1}\right) \rightarrow X_{1}$, $i_{2}: T_{2}=T\left(X_{2}\right) \rightarrow X_{2}$, and corresponding Weyl groups $W_{1}=W\left(X_{1}\right), W_{2}=W\left(X_{2}\right)$, respectively. Write $\operatorname{Rep}\left(X_{1}, X_{2}\right)=\left[\mathrm{B} X_{1}, \mathrm{~B} X_{2}\right]$ for the set of conjugacy classes of morphisms $X_{1} \rightarrow X_{2}$. The restriction map

$$
\operatorname{Rep}\left(X_{1}, X_{2}\right) \stackrel{\overline{i_{1}}}{\longrightarrow} \operatorname{Rep}\left(T_{1}, X_{2}\right) \stackrel{\stackrel{i_{2}}{\cong}}{\cong} W_{2} \backslash \operatorname{Rep}\left(T_{1}, T_{2}\right)
$$

where $\overline{i_{1}}$ and the bijection $\underline{i}_{2}$ [24, 3.4.(1)] are induced by $i_{1}$ and $i_{2}$ respectively, takes $f \in \operatorname{Rep}\left(X_{1}, X_{2}\right)$ to $f \mid \bar{T}_{1}=W_{2} T(f)$ where $T(f)$ is any lift

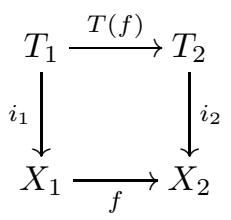

of $f$ to a morphism between the maximal tori.

It is sometimes useful to know that certain morphisms $T_{1} \rightarrow T_{2}$ are not of the form $T(f)$ for any $f: X_{1} \rightarrow X_{2}$.

Lemma 2.1 (Cf. [19 1.8]). Let $f: X_{1} \rightarrow X_{2}$ be a p-compact group morphism where $p$ is odd and $X_{1}$ is connected. Assume that

- $\pi_{1}(T(f))$ is injective, and

- $p$ divides the order of the Weyl group $W_{1}$.

Then $p$ does not divide $\pi_{1}(T(f))$ in $\operatorname{Hom}\left(\pi_{1}\left(T_{1}\right), \pi_{1}\left(T_{2}\right)\right)$. 
Proof. By fixed point theory [14, 2.10, 2.14], $f$ lifts to a morphism of the $p$ normalizers $N_{p}(f): \operatorname{Syl}_{p}\left(N_{1}\right) \rightarrow \operatorname{Syl}_{p}\left(N_{2}\right)$. The assumption that $\pi_{1}(T(f))$ be injective implies, since $W_{1}$ is faithfully represented in $\pi_{1}\left(T_{1}\right)$ [13, 9.7], that $\pi_{0}\left(N_{p}(f)\right)$ embeds the Sylow $p$-subgroup of $W_{1}$ into $W_{2}$.

Choose a monomorphism $\mu: \mathbf{Z} / p \rightarrow \operatorname{Syl}_{p}\left(N_{1}\right)$ such that $\pi_{0}(\mu): \mathbf{Z} / p \rightarrow \operatorname{Syl}_{p}\left(W_{1}\right)$ is also injective. This is possible since the epimorphism $\operatorname{Syl}_{p}\left(N_{1}\right) \rightarrow \operatorname{Syl}_{p}\left(W_{1}\right)$ admits a section when $p$ is odd [4. Note that the composition $N_{p}(f) \mu$ is a monomorphism since it induces a monomorphism on component groups. Consider now the commutative diagram

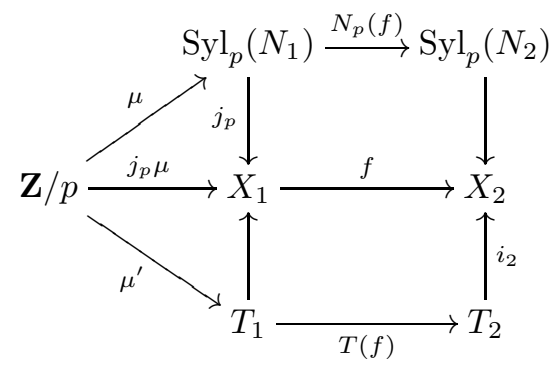

where $\mu^{\prime}$ is a lift of $j_{p} \mu[13,4.7,5.6]$. Since $N_{p}(f) \mu$ is monomorphic, so is $i_{2} T(f) \mu^{\prime}$ by commutativity of the diagram. However, this map would be trivial if $\pi_{1}(T(f))$ were divisible by $p$.

This lemma is also true for $p=2$ provided the extra assumption

- there exists a monomorphism $\mathbf{Z} / 2 \rightarrow \operatorname{Syl}_{2}\left(W_{1}\right)$ that factors through $\operatorname{Syl}_{2}\left(N_{1}\right)$ is added.

We now return to the general situation of (2.1). For any element $w_{1}$ of the Weyl group $W_{1}$ of $X_{1}$,

$$
\underline{i}_{2}\left(T(f) \circ w_{1}\right)=i_{2} \circ T(f) \circ w_{1}=f \circ i_{1} \circ w_{1}=f \circ i_{1}=i_{2} \circ T(f)=\underline{i}_{2}(T(f))
$$

so there must exist some Weyl group element $w_{2} \in W_{2}$ such that $T(f) \circ w_{1}=$ $w_{2} \circ T(f)$. Thus the lift $T(f)$ lies in the subset $W_{2} \backslash \operatorname{Adm}\left(T_{1}, T_{2}\right)$ where

Definition 2.2. $\operatorname{Adm}\left(T_{1}, T_{2}\right)=\left\{a \in \operatorname{Rep}\left(T_{1}, T_{2}\right) \mid a \cdot W_{1} \subseteq W_{2} \cdot a\right\}$.

The fundamental group functor can be used to identify this set $\operatorname{Adm}\left(T_{1}, T_{2}\right)$ of admissible representations with the corresponding set $\operatorname{Adm}\left(\pi_{1}\left(T_{1}\right), \pi_{1}\left(T_{2}\right)\right)$ $\subseteq \operatorname{Hom}\left(\pi_{1}\left(T_{1}\right), \pi_{1}\left(T_{2}\right)\right)$ of admissible homomorphisms; that is, homomorphisms $a: \pi_{1}\left(T_{1}\right) \rightarrow \pi_{1}\left(T_{2}\right)$ such that $a \cdot W_{1} \subseteq W_{2} \cdot a$.

We shall now determine the admissible homomorphisms in the particular case where $p=3, X_{1}=\mathrm{DI}_{2}\left[23,3\right.$, and $X_{2}=\mathrm{F}_{4}$ viewed as a 3-compact group.

The construction of $\mathrm{BDI}_{2}$ exhibits monomorphisms 23 .

$$
T_{1} \rightarrow \mathrm{SU}(3) \rightarrow \mathrm{DI}_{2}
$$

of maximal rank where the morphism $T_{1}=T(\mathrm{SU}(3)) \rightarrow \mathrm{SU}(3)$ is a maximal torus for $\mathrm{SU}(3)$. Take the composite morphism (2.2) as the maximal torus for $\mathrm{DI}_{2}$.

Let $i_{2}: T_{2}=T\left(\mathrm{~F}_{4}\right) \rightarrow \mathrm{F}_{4}$ be a maximal torus for the simple compact Lie group $\mathrm{F}_{4}$ (defining a maximal torus for the corresponding 3-compact group).

There is a standard identification

$$
\pi_{1}\left(T_{1}\right) \stackrel{\cong}{\longrightarrow} \Sigma_{0}\left(\mathbf{Z}_{3}^{3}\right)=\left\{\left(x_{1}, x_{2}, x_{3}\right) \in \mathbf{Z}_{3}^{3} \mid x_{1}+x_{2}+x_{3}=0\right\}
$$


where $\mathbf{Z}_{3}$ stands for the ring of 3-adic integers. We shall use $\{(1,-1,0),(0,1,-1)\}$ as a basis for the free $\mathbf{Z}_{3}$-module $\Sigma_{0}\left(\mathbf{Z}_{3}{ }^{3}\right)$ thus identifying $\Sigma_{0}\left(\mathbf{Z}_{3}{ }^{3}\right)$ with $\mathbf{Z}_{3}{ }^{2}$. Under this identification we may assume [31 7.3, Example 1] 23] that the Weyl group $W_{1}=W\left(\mathrm{DI}_{2}\right)$ of $\mathrm{DI}_{2}$ corresponds to the subgroup

$$
W\left(\mathrm{DI}_{2}\right)=\langle\alpha, \sigma, \tau\rangle \subseteq \operatorname{Aut}\left(\Sigma_{0}\left(\mathbf{Z}_{3}^{3}\right)\right)=\operatorname{GL}\left(2, \mathbf{Z}_{3}\right)
$$

with generators

$$
\alpha=\frac{1}{1+\zeta}\left(\begin{array}{cc}
-1+\zeta & -\zeta \\
\zeta & 1-\zeta
\end{array}\right), \quad \sigma=\left(\begin{array}{cc}
0 & -1 \\
1 & -1
\end{array}\right), \quad \tau=\left(\begin{array}{rr}
0 & -1 \\
-1 & 0
\end{array}\right)
$$

where $\zeta^{2}+2=0$ and $\zeta \equiv 1 \bmod 3$. The subgroup $W(\mathrm{SU}(3))=\langle\sigma, \tau\rangle \cong \Sigma_{3}$ is the Weyl group of $\mathrm{SU}(3)$.

Similarly, there is a standard identification [7] between the fundamental group $\pi_{1}\left(T_{2}\right)$ of the maximal torus for $\mathrm{F}_{4}$ and the free abelian group

$$
\Sigma_{2}\left(\mathbf{Z}^{4}\right)=\left\{\left(x_{1}, x_{2}, x_{3}, x_{4}\right) \in \mathbf{Z}^{4} \mid x_{1}+x_{2}+x_{3}+x_{4} \in 2 \mathbf{Z}\right\} .
$$

Under this identification, the Weyl group $W_{2}=W\left(\mathrm{~F}_{4}\right)$ of $\mathrm{F}_{4}$ is carried to the group (of order $1152=384 \cdot 3$ )

$$
W\left(\mathrm{~F}_{4}\right)=W\left(\mathrm{~B}_{4}\right) E \cup W\left(\mathrm{~B}_{4}\right) H_{1} \cup W\left(\mathrm{~B}_{4}\right) H_{2}
$$

where $W\left(\mathrm{~B}_{4}\right)$ is the reflection group (of order $384=2^{4} \cdot 4$ !) of all signed permutation matrices, and $H_{1}$ and $H_{2}$ are the matrices

$$
H_{1}=\frac{1}{2}\left(\begin{array}{rrrr}
-1 & 1 & 1 & 1 \\
1 & -1 & 1 & 1 \\
1 & 1 & -1 & 1 \\
1 & 1 & 1 & -1
\end{array}\right), \quad H_{2}=\frac{1}{2}\left(\begin{array}{rrrr}
1 & 1 & 1 & 1 \\
1 & 1 & -1 & -1 \\
1 & -1 & 1 & -1 \\
1 & -1 & -1 & 1
\end{array}\right) .
$$

Note that the group $W\left(\mathrm{~B}_{4}\right)$ and the matrices $H_{1}$ and $H_{2}$ preserve the submodule $\Sigma_{2}\left(\mathbf{Z}^{4}\right)$ of $\mathbf{Z}^{4}$. The operators $H_{1}$ and $H_{2}$ satisfy the relations $H_{1}^{2}=E=$ $H_{2}^{2}, H_{2} H_{1}=-H_{2}, H_{1} H_{2}=\operatorname{diag}(-1,1,1,1) H_{1}$. For $\mathrm{F}_{4}$ viewed as a 3 -compact group with maximal torus $T_{2}$, extensions of scalars provide an identification

$$
\pi_{1}\left(T_{2}\right) \stackrel{\cong}{\longrightarrow} \Sigma_{2}\left(\mathbf{Z}^{4}\right) \otimes \mathbf{Z}_{3} \stackrel{\cong}{\longrightarrow} \mathbf{Z}^{4} \otimes \mathbf{Z}_{3} \cong \mathbf{Z}_{3}^{4}
$$

taking the Weyl group $W\left(\mathrm{~F}_{4}\right)$ onto the reflection group $W\left(\mathrm{~F}_{4}\right)<\mathrm{GL}\left(4, \mathbf{Z}_{3}\right)$ as defined in (2.3).

The linear map $A(v): \Sigma_{0}\left(\mathbf{Z}_{3}{ }^{3}\right) \rightarrow \mathbf{Z}_{3}{ }^{4}$ with matrix

$$
A(v)=v \cdot\left(\begin{array}{rc}
-\zeta & 1 \\
\zeta & 1-\zeta \\
0 & 1+\zeta \\
-2 & 1
\end{array}\right), \quad v \in \mathbf{Z}_{3}
$$

is admissible with respect to $W\left(\mathrm{DI}_{2}\right)$ and $W\left(\mathrm{~F}_{4}\right)$ since $A(v) w=\chi(w) A(v)$ for all $w \in W\left(\mathrm{DI}_{2}\right)$ where $\chi: W\left(\mathrm{DI}_{2}\right) \rightarrow W\left(\mathrm{~F}_{4}\right)$ is the group homomorphism with the values

$$
\left(\begin{array}{rrrr}
0 & 0 & 0 & -1 \\
0 & 0 & -1 & 0 \\
0 & 1 & 0 & 0 \\
1 & 0 & 0 & 0
\end{array}\right), \quad \frac{1}{2}\left(\begin{array}{rrrr}
-1 & -1 & 1 & -1 \\
1 & -1 & -1 & -1 \\
-1 & 1 & -1 & -1 \\
1 & 1 & 1 & -1
\end{array}\right), \quad \frac{1}{2}\left(\begin{array}{rrrr}
-1 & -1 & 1 & 1 \\
-1 & 1 & -1 & 1 \\
1 & -1 & -1 & 1 \\
1 & 1 & 1 & 1
\end{array}\right)
$$

on the generators $\alpha, \sigma$, and $\tau$, respectively. 
Note that $A(v)$ and $-A(v)$ lie in the same orbit under the action of $W\left(\mathrm{~F}_{4}\right)$ as $-E \in W\left(\mathrm{~F}_{4}\right)$.

Lemma 2.3. Let $v$ be a 3-adic integer.

1. The linear homomorphism $A: \Sigma_{0}\left(\mathbf{Z}_{3}{ }^{3}\right) \rightarrow \mathbf{Z}_{3}{ }^{4}$ is admissible with respect to $W\left(\mathrm{DI}_{2}\right)$ and $W\left(\mathrm{~F}_{4}\right)$ if and only if $A \in W\left(\mathrm{~F}_{4}\right) A(v)$ for some 3-adic integer $v \in \mathbf{Z}_{3}$.

2. The linear map $A(v)$ is split injective if and only if $v \in \mathbf{Z}_{3}{ }^{*}$ is a 3-adic unit.

3. The map

$$
\begin{aligned}
\{ \pm 1\} \backslash \mathbf{Z}_{3}{ }^{*} & \rightarrow W\left(\mathrm{~F}_{4}\right) \backslash \operatorname{Hom}_{\mathbf{Z}_{3}}\left(\Sigma_{0}\left(\mathbf{Z}_{3}{ }^{3}\right), \mathbf{Z}_{3}{ }^{4}\right) \\
\pm v & \rightarrow W\left(\mathrm{~F}_{4}\right) A(v)
\end{aligned}
$$

is injective.

The proof, which we omit, is by direct (computer assisted) computation.

\section{Constructing maps out of $\mathrm{BDI}_{2}$}

Let $\mathbf{I}=\mathbf{I}\left(W\left(\mathrm{DI}_{2}\right), W(\mathrm{SU}(3))\right)$ be the category with two objects, 0 and 1 , and morphism sets $\mathbf{I}(0,0)=N_{W\left(\mathrm{DI}_{2}\right)}(W(\mathrm{SU}(3)) / W(\mathrm{SU}(3))) \cong Z\left(W\left(\mathrm{DI}_{2}\right)\right) \cong \mathbf{Z} / 2$, $\mathbf{I}(1,1)=W\left(\mathrm{DI}_{2}\right), \mathbf{I}(0,1)=W\left(\mathrm{DI}_{2}\right) / W(\mathrm{SU}(3))$, and $\mathbf{I}(1,0)=\emptyset$.

The space $\mathrm{BDI}_{2}$ can be constructed [23, 6.10] as the homotopy colimit of the $\mathbf{I}^{\mathrm{op}}$-space

$$
(\mathbf{z} / 2)^{\mathrm{op}} C \mathrm{BSU}(3) \stackrel{W(\mathrm{SU}(3))^{\mathrm{op}} \backslash W\left(\mathrm{DI}_{2}\right)^{\mathrm{op}}}{\longleftarrow} \mathrm{B} T \frown W\left(\mathrm{DI}_{2}\right)^{\mathrm{op}}
$$

where $\mathbf{Z} / 2$ acts on $\mathrm{B} \mathrm{SU}(3)$ as $\left\{\psi^{ \pm 1}\right\}$. We shall use diagram (3.1) in connection with Wojtkowiak obstruction theory 37. to prove existence and uniqueness of certain maps out of $\mathrm{BDI}_{2}$.

The set of conjugacy classes of monomorphisms, $\mathrm{Mono}\left(\mathrm{DI}_{2}, \mathrm{~F}_{4}\right)$ of monomorphisms $\mathrm{DI}_{2} \longrightarrow \mathrm{F}_{4}$ will turn out to be faithfully represented in the set Mono(SU(3), $\left.\mathrm{F}_{4}\right)$ of conjugacy classes of monomorphisms SU(3) $\hookrightarrow \mathrm{F}_{4}$ which we now describe [25]:

There exists a bijection

$$
\begin{aligned}
\left\{(u, v) \in\left(\mathbf{Z}_{3}\right)^{2} \mid u+v \equiv 1 \bmod 3\right\} & \rightarrow \operatorname{Mono}\left(\mathrm{SU}(3), \mathrm{F}_{4}\right) \\
(u, v) & \rightarrow e \psi^{(u, v)}
\end{aligned}
$$

where $\psi^{(u, v)}$ is the composite morphism

$$
\mathrm{SU}(3) \stackrel{\Delta}{\longrightarrow} \mathrm{SU}(3) \times \mathrm{SU}(3) \stackrel{\psi^{u} \times \psi^{v}}{\longrightarrow} \mathrm{SU}(3) \times \mathrm{SU}(3) \rightarrow \mathrm{SU}(3,3)
$$

and $e: \mathrm{SU}(3,3) \rightarrow \mathrm{F}_{4}$ is the inclusion, described in [19, 3.3], of $\mathrm{SU}(3,3)=\mathrm{SU}(3) \times \mathbf{z} / 3$ $\mathrm{SU}(3)$ as a maximal rank subgroup of $\mathrm{F}_{4}$. (The central $\mathbf{Z} / 3$ in $\mathrm{SU}(3) \times \mathrm{SU}(3)$ is generated by $\left(z E, z^{-1} E\right)$ where $z \neq 1$ is a third root of unity.) Furthermore, the injective restriction map

$$
\operatorname{Mono}\left(\mathrm{SU}(3), \mathrm{F}_{4}\right) \rightarrow \operatorname{Mono}\left(T(\mathrm{SU}(3)), \mathrm{F}_{4}\right)=W\left(\mathrm{~F}_{4}\right) \backslash \operatorname{Hom}_{\mathbf{Z}_{3}}\left(\Sigma_{0}\left(\mathbf{Z}_{3}{ }^{3}\right), \mathbf{Z}_{3}{ }^{4}\right)
$$

takes the monomorphism $e \psi^{(u, v)}$ to

$$
W\left(\mathrm{~F}_{4}\right)\left(\begin{array}{cc}
-u & v \\
u & -u+v \\
0 & u+v \\
-2 v & v
\end{array}\right)
$$


Lemma 3.1. Let $f \in \operatorname{Mono}\left(\mathrm{DI}_{2}, \mathrm{~F}_{4}\right)$ be a monomorphism. Then the restriction to $\mathrm{SU}(3)$,

$$
\left.f\right|_{\mathrm{SU}(3)}=e \psi^{(\zeta v, v)}
$$

for a uniquely determined 3-adic unit $v \in \mathbf{Z}_{3}{ }^{*}, v \equiv-1 \bmod 3$.

Proof. According to (2.3), the restriction of $f$ to the maximal torus,

$$
f \mid T\left(\mathrm{DI}_{2}\right) \in \operatorname{Mono}\left(T\left(\mathrm{DI}_{2}\right), \mathrm{F}_{4}\right)=W\left(\mathrm{~F}_{4}\right) \backslash \operatorname{Hom}_{\mathbf{Z}_{3}}\left(\Sigma_{0}\left(\mathbf{Z}_{3}{ }^{3}\right), \mathbf{Z}_{3}{ }^{4}\right)
$$

is of the form $f \mid T\left(\mathrm{DI}_{2}\right)=W\left(\mathrm{~F}_{4}\right) A(v)$ for some 3-adic unit $v \in \mathbf{Z}_{3}{ }^{*}$, uniquely determined up to sign. (The 3 -adic number $v$ must be a unit because $A(v)$ is split injective as $f \mid T\left(\mathrm{DI}_{2}\right)$ is a monomorphism [26, 23].) But this means that the restriction $f \mid \mathrm{SU}(3)=e \psi^{(\zeta v, v)}$ for a uniquely determined 3-adic unit $v \in \mathbf{Z}_{3}{ }^{*}$, $v \equiv-1 \bmod 3$.

Let now $v \in \mathbf{Z}_{3}{ }^{*}$ be any 3 -adic unit such that $v \equiv-1 \bmod 3$. Then the two homotopy classes $e \psi^{(\zeta v, v)} \in\left[\mathrm{BSU}(3), \mathrm{BF}_{4}\right]$ and $W\left(\mathrm{~F}_{4}\right) A(v) \in\left[\mathrm{BT}(\mathrm{SU}(3)), \mathrm{BF}_{4}\right]$ form a homotopy coherent pair of maps out of the $\mathbf{I}^{\mathrm{op}}$-space (3.1) in the sense that

- $e \psi^{(\zeta v, v)} \mid T(\mathrm{SU}(3))=W\left(\mathrm{~F}_{4}\right) A(v)$,

- $W\left(\mathrm{~F}_{4}\right) A(v)$ is $W\left(\mathrm{DI}_{2}\right)$-invariant,

- $e \psi^{(\zeta v, v)}$ is $\left\langle\psi^{-1}\right\rangle$-invariant,

where the last property follows from the computation

$$
e \psi^{(\zeta v, v)} \psi^{-1}=e\left(\psi^{-1} \times \psi^{-1}\right) \psi^{(\zeta v, v)}=e \psi^{(\zeta v, v)}
$$

which uses the identity $e\left(\psi^{-1} \times \psi^{-1}\right)=e$ from [19, 3.3]. Therefore, there is an induced I-space

$$
\mathrm{z} / 2 \mathrm{~B} C_{\mathrm{F}_{4}}\left(e \psi^{(\zeta v, v)}\right) \stackrel{W\left(\mathrm{DI}_{2}\right) / W(\mathrm{SU}(3))}{\longrightarrow} \mathrm{B} C_{\mathrm{F}_{4}}\left(W\left(\mathrm{~F}_{4}\right) A(v)\right) \supseteq W\left(\mathrm{DI}_{2}\right)
$$

of connected mapping spaces where

$$
\begin{gathered}
\mathrm{B} C_{\mathrm{F}_{4}}\left(e \psi^{(\zeta v, v)}\right)=\operatorname{map}\left(\mathrm{BSU}(3), \mathrm{BF}_{4}\right)_{\mathrm{Be} \psi(\zeta v, v)} \simeq \mathrm{B} Z(\mathrm{SU}(3)), \\
\mathrm{B} C_{\mathrm{F}_{4}}\left(W\left(\mathrm{~F}_{4}\right) A(v)\right) \operatorname{map}\left(\mathrm{B} T(\mathrm{SU}(3)), \mathrm{BF}_{4}\right)_{\mathrm{B}\left(W\left(\mathrm{~F}_{4}\right) A(v)\right)} \simeq \mathrm{B} T\left(\mathrm{~F}_{4}\right)
\end{gathered}
$$

as $e \psi^{(\zeta v, v)}$ is centric and $p$-toric [25]. These two spaces are simple so we may apply the homotopy functor $\pi_{t}$ to (3.4) to obtain the $\mathbf{I}$-module $\underline{\pi}_{t}(v)$.

Lemma 3.2. $\lim _{\mathbf{I}}^{-s} \underline{\pi}_{t}(v)=0$ for all $s \leq 0, t \geq 0, s+t \geq-1$ and for all 3-adic units $v \in \mathbf{Z}_{3}{ }^{*}$ with $v \equiv-1 \bmod 3$.

Proof. For the I-module $\underline{\pi}_{1}(v)$,

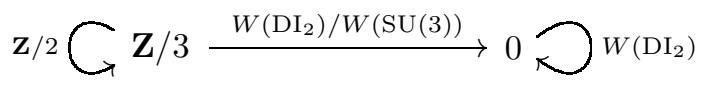

we have $\lim _{\mathbf{I}}^{-s} \underline{\pi}_{1}(v)=H^{-s}(\mathbf{Z} / 2 ; \mathbf{Z} / 3)$ which is trivial since $\mathbf{Z} / 2=\left\langle\psi^{-1}\right\rangle$ acts non-trivially on the center $\mathbf{Z} / 3=Z(\mathrm{SU}(3))$. For the $\mathbf{I}$-module $\underline{\pi}_{2}(v)$,

$$
\mathbf{Z} / 2 \bigcirc 0 \stackrel{W\left(\mathrm{DI}_{2}\right) / W(\mathrm{SU}(3))}{\longrightarrow} \mathbf{Z}_{3}^{4} \bigcirc W\left(\mathrm{DI}_{2}\right)
$$

we have $\lim _{\mathbf{I}}^{0} \underline{\pi}_{2}(v)=0, \lim _{\mathbf{I}}^{1} \underline{\pi}_{2}(v)=\left(\mathbf{Z}_{3}^{4}\right)^{Z\left(W\left(\mathrm{DI}_{2}\right) \times W(\mathrm{SU}(3))\right.}=0$ as $-E$ belongs to the center $Z\left(W\left(\mathrm{DI}_{2}\right)\right)$, and $\lim _{\mathbf{I}}^{-s} \underline{\pi}_{2}(v)=0$ for $s \leq-2$ by [23, 12.7].

With this lemma in place we are ready for one of the main results of this paper. 
Theorem 3.3. The following hold for the set $\mathrm{Mono}\left(\mathrm{DI}_{2}, \mathrm{~F}_{4}\right)$ of conjugacy classes of monomorphisms $\mathrm{DI}_{2} \rightarrow \mathrm{F}_{4}$ :

1. The restriction map

$$
\operatorname{Mono}\left(\mathrm{DI}_{2}, \mathrm{~F}_{4}\right) \rightarrow \operatorname{Mono}\left(\mathrm{SU}(3), \mathrm{F}_{4}\right)
$$

is an injection with $\left\{e \psi^{(\zeta v, v)} \mid v \in \mathbf{Z}_{3}{ }^{*}, v \equiv-1 \bmod 3\right\}$ as its image.

2. The restriction map

$$
\operatorname{Mono}\left(\mathrm{DI}_{2}, \mathrm{~F}_{4}\right) \rightarrow \operatorname{Mono}\left(T\left(\mathrm{DI}_{2}\right), \mathrm{F}_{4}\right)
$$

is an injection with $\left\{W\left(\mathrm{~F}_{4}\right) A(v) \mid v \in \mathbf{Z}_{3}{ }^{*}, v \equiv-1 \bmod 3\right\}$ as its image.

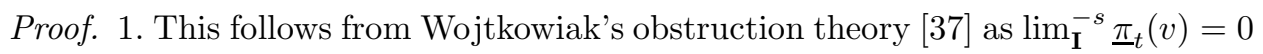
when $s+t=0$ and $s+t=-1$.

2. The map is a composition

$$
\operatorname{Mono}\left(\mathrm{DI}_{2}, \mathrm{~F}_{4}\right) \rightarrow \operatorname{Mono}\left(\mathrm{SU}(3), \mathrm{F}_{4}\right) \rightarrow \operatorname{Mono}\left(T(\mathrm{SU}(3)), \mathrm{F}_{4}\right)
$$

of two injections (3.2).

Let $f(v): \mathrm{DI}_{2} \rightarrow \mathrm{F}_{4}, v \in \mathbf{Z}_{3}{ }^{*}, v \equiv-1 \bmod 3$, denote the unique extension to $\mathrm{DI}_{2}$ of the monomorphism $e \psi^{(\zeta v, v)}: \mathrm{SU}(3) \rightarrow \mathrm{F}_{4}$ on $\mathrm{SU}(3)$.

Corollary 3.4. 1. The group $\operatorname{Out}\left(\mathrm{DI}_{2}\right)$ acts simply transitively on the set Mono $\left(\mathrm{DI}_{2}, \mathrm{~F}_{4}\right)$ of monomorphisms.

2. The centralizer of any monomorphism $\mathrm{DI}_{2} \rightarrow \mathrm{F}_{4}$ is trivial.

3. The Weyl group [23, 9.5] of any monomorphism $\mathrm{DI}_{2} \rightarrow \mathrm{F}_{4}$ is trivial.

Proof. 1. This is clear since [23] $\operatorname{Out}\left(\mathrm{DI}_{2}\right) \cong\left\{v \in \mathbf{Z}_{3}{ }^{*} \mid v \equiv 1 \bmod 3\right\}$ and

$$
f(v) \psi^{u}=e \psi^{(\zeta v, v)} \psi^{u}=e \psi^{(\zeta v u, v u)}=f(v u)
$$

so that $f(v) \psi^{u}=f(v) \Leftrightarrow u=1$.

2 . The $E_{2}$-page of the Bousfield-Kan spectral sequence, $E_{s}^{s t}=\lim _{\mathbf{I}}^{-s} \underline{\pi}_{t}(v)$, converging to $\pi_{s+t}\left(\mathrm{BC}_{\mathrm{F}_{4}}\left(f(v) \mathrm{DI}_{2}\right)\right)$ vanishes completely (3.2).

3. The Weyl group $W_{\mathrm{F}_{4}}\left(f(v) \mathrm{DI}_{2}\right)$ is $[23,9.6]$ the stabilizer subgroup for $\operatorname{Out}\left(\mathrm{DI}_{2}\right)$ acting on $f(v) \in \operatorname{Mono}\left(\mathrm{DI}_{2}, \mathrm{~F}_{4}\right)$. We have just seen that this stabilizer is trivial.

In a way, (3.41) says that $\mathrm{F}_{4}$ contains a unique copy of $\mathrm{DI}_{2}$.

Finally, we determine the set $\operatorname{Rep}\left(\mathrm{DI}_{2}, \mathrm{~F}_{4}\right)=\left[\mathrm{BDI}_{2}, \mathrm{BF}_{4}\right]$ of all maps up to homotopy.

Proposition 3.6. Any non-trivial morphism of $\mathrm{DI}_{2}$ to $\mathrm{F}_{4}$ is a monomorphism.

Proof. Let $f: \mathrm{DI}_{2} \rightarrow \mathrm{F}_{4}$ be any non-trivial morphism. Then $f \mid T\left(\mathrm{DI}_{2}\right)=W\left(\mathrm{~F}_{4}\right) A(v)$ $\in\left[\mathrm{BT}\left(\mathrm{DI}_{2}\right), \mathrm{BF}_{4}\right]$ for some 3-adic integer $v \in \mathbf{Z}_{3}$. This 3-adic integer, $v$, must be non-zero as $f$ is non-trivial [24, 6.7] and even a 3 -adic unit by (2.1). This shows that $A(v)$ is split injective and hence [26, 3.6] [23, 8.2] that $f$ is a monomorphism.

Proof of Theorem 1.1. We conclude from (3.3, 3.4, 3.6) that

$$
\operatorname{Rep}\left(\mathrm{DI}_{2}, \mathrm{~F}_{4}\right)=\{0\} \cup \operatorname{Mono}\left(\mathrm{DI}_{2}, \mathrm{~F}_{4}\right)
$$

is in bijection with the set $\{0\} \cup \mathbf{Z}_{3}{ }^{*} /\{ \pm 1\}$.

In the following, we let $\mathrm{B} \alpha: \mathrm{BDI}_{2} \rightarrow \mathrm{BF}_{4}$ denote the monomorphism $B f(-1)$ corresponding (3.3) to the admissible homomorphism with matrix $A(-1)(2.4)$. 
4. The homogeneous SPace $\mathrm{F}_{4} / \mathrm{DI}_{2}$

In this section we compute the mod 3 cohomology of the exotic homogeneous space $\mathrm{F}_{4} / \mathrm{DI}_{2}$.

The unstable mod 3 cohomology of $\mathrm{F}_{4}$ is known since Borel [6]:

$$
H^{*}\left(\mathrm{~F}_{4} ; \mathbf{F}_{3}\right) \cong E\left[z_{3}, z_{7}, z_{11}, z_{15}\right] \otimes P\left[z_{8}\right] /\left(z_{8}{ }^{3}\right)
$$

with $P^{1} z_{3}=z_{7}, \beta z_{7}=z_{8}$ and $P^{1} z_{11}=z_{15}$.

The 3 -complete space $\mathrm{BDI}_{2}$ realizes the rank 2 mod 3 Dickson algebra meaning that

$$
H^{*}\left(\mathrm{BDI}_{2} ; \mathbf{F}_{3}\right) \cong P\left[x_{12}, x_{16}\right]
$$

with $P^{1} x_{12}=x_{16}$.

The homogeneous space $\mathrm{F}_{4} / \mathrm{DI}_{2}$ is defined as the homotopy fibre of the map $\mathrm{BDI}_{2} \stackrel{\mathrm{B} \alpha}{\longrightarrow} \mathrm{BF}_{4}$, and we have a sequence of fibrations

$$
\mathrm{DI}_{2} \stackrel{\Omega \mathrm{B} \alpha}{\longrightarrow} \mathrm{F}_{4} \stackrel{\pi}{\longrightarrow} \mathrm{F}_{4} / \mathrm{DI}_{2} \stackrel{j}{\longrightarrow} \mathrm{BDI}_{2} \stackrel{\mathrm{B} \alpha}{\longrightarrow} \mathrm{BF}_{4} .
$$

We now investigate the value of the functor $H^{*}\left(-; \mathbf{F}_{3}\right)$ on this sequence.

Proposition 4.1. The effects on mod 3 cohomology of the maps $\pi$ and $j$ of (4.1) are as follows:

1. The unstable mod 3 cohomology algebra of $\mathrm{F}_{4} / \mathrm{DI}_{2}$ is

$$
H^{*}\left(\mathrm{~F}_{4} / \mathrm{DI}_{2} ; \mathbf{F}_{3}\right) \cong E\left[z_{3}, z_{7}\right] \otimes P\left[z_{8}\right] /\left(z_{8}^{3}\right)
$$

with $P^{1} z_{3}=z_{7}$ and $\beta z_{7}=z_{8}$.

2. $H^{*}\left(\pi ; \mathbf{F}_{3}\right)$ is the obvious inclusion

$$
\begin{aligned}
H^{*}\left(\mathrm{~F}_{4} / \mathrm{DI}_{2} ; \mathbf{F}_{3}\right) & \cong E\left[z_{3}, z_{7}\right] \otimes P\left[z_{8}\right] /\left(z_{8}^{3}\right) \\
& \subseteq E\left[z_{3}, z_{7}, z_{11}, z_{15}\right] \otimes P\left[z_{8}\right] /\left(z_{8}^{3}\right) \cong H^{*}\left(\mathrm{~F}_{4} ; \mathbf{F}_{3}\right)
\end{aligned}
$$

of algebras.

3. $H^{>0}\left(j ; \mathbf{F}_{3}\right)$ is the trivial homomorphism $H^{>0}\left(\mathrm{BDI}_{2} ; \mathbf{F}_{3}\right) \rightarrow H^{>0}\left(\mathrm{~F}_{4} / \mathrm{DI}_{2} ; \mathbf{F}_{3}\right)$.

Proof. It consists of a calculation with the Serre spectral sequence for the fibration $\mathrm{F}_{4} \stackrel{\pi}{\longrightarrow} \mathrm{F}_{4} / \mathrm{DI}_{2} \stackrel{j}{\rightarrow} \mathrm{BDI}_{2}$. This starts at $E_{2}^{p, q} \cong H^{p}\left(\mathrm{BDI}_{2} ; \mathbf{F}_{3}\right) \otimes H^{q}\left(\mathrm{~F}_{4} ; \mathbf{F}_{3}\right)$.

For degree reasons the classes $z_{3}, z_{7}$ and $z_{8}$ in the vertical edge are permanent cycles. Assume that $z_{11} \in E_{2}^{0, *}$ is a permanent cycle. Then $z_{15}=P^{1} z_{11}$ is also a permanent cycle and therefore the spectral sequence collapses at $E_{2}$. But this is impossible because the fibre $\mathrm{F}_{4} / \mathrm{DI}_{2}$ of the monomorphism $\mathrm{B} \alpha$ is $\mathbf{F}_{3}$-finite [13.

Hence, the class $z_{11}$ transgresses to $\pm x_{12} \in H^{*}\left(\mathrm{BDI}_{2} ; \mathbf{F}_{3}\right) \cong E_{2}^{*, 0}$ and then $z_{15}=P^{1} z_{11}$ trangresses to $\pm x_{16}=P^{1}\left( \pm x_{12}\right)$. After this last, the spectral sequence collapses to $E_{\infty}^{* * *} \cong E_{\infty}^{0, *} \cong E\left[z_{3}, z_{7}\right] \otimes P\left[z_{8}\right] /\left(z_{8}{ }^{3}\right)$ and the edge homomorphisms are an injection

$$
H^{*}\left(\mathrm{~F}_{4} / \mathrm{DI}_{2} ; \mathbf{F}_{3}\right) \cong E_{\infty}^{0, *} \cong E\left[z_{3}, z_{7}\right] \otimes P\left[z_{8}\right] /\left(z_{8}{ }^{3}\right) \longmapsto E_{2}^{0, *} \cong H^{*}\left(\mathrm{~F}_{4} ; \mathbf{F}_{3}\right)
$$

and the trivial homomorphism

$$
H^{*}\left(\mathrm{BDI}_{2} ; \mathbf{F}_{3}\right) \cong E_{2}^{*, 0} \longmapsto E_{\infty}^{0, *} \cong \mathbf{F}_{3} \subset H^{*}\left(\mathrm{~F}_{4} / \mathrm{DI}_{2} ; \mathbf{F}_{3}\right)
$$

which proves the proposition. 
Remark 4.2. The mod 3 cohomology of $\mathrm{F}_{4} / \mathrm{DI}_{2}$ coincides with that of Harper's molecule [17] at the prime 3 and this implies that they are actually homotopy equivalent up to 3 -completion. Details will be worked out in Section 7

\section{FRIEDLANDER'S EXCEPTIONAL ISOGENY}

In [15], E.M. Friedlander showed the existence of a self-homotopy equivalence $\mathrm{B} \varphi$ of $\left(\mathrm{BF}_{4}\right)_{1 / 2}$ that restricts to the maximal torus to the isogeny

$$
\mathrm{B} T(\varphi): \mathrm{B} T\left(\mathrm{~F}_{4}\right) \rightarrow \mathrm{B} T\left(\mathrm{~F}_{4}\right)
$$

determined by the matrix

$$
\pi_{2}(\mathrm{~B} T(\varphi))=\left(\begin{array}{cccc}
1 & -1 & 0 & 0 \\
1 & 1 & 0 & 0 \\
0 & 0 & 1 & -1 \\
0 & 0 & 1 & 1
\end{array}\right)
$$

acting on $\pi_{2}\left(\mathrm{~B} T\left(\mathrm{~F}_{4}\right)\right) \cong \mathbf{Z}_{3}{ }^{4}$. The automorphism $\mathrm{B} \varphi$ of the 3-compact group $\mathrm{BF}_{4}$ satisfies the relation $\mathrm{B} \varphi \circ \mathrm{B} \varphi \simeq \mathrm{B} \psi^{2}$. To see this, note that $W\left(\mathrm{~F}_{4}\right) T(\varphi)^{2}=$ $W\left(\mathrm{~F}_{4}\right) T\left(\psi^{2}\right)$ and recall that $\mathrm{BF}_{4}$ has $N$-determined automorphisms; that is, restriction to the maximal torus normalizer provides a bijective map $\operatorname{Out}\left(\mathrm{F}_{4}\right) \rightarrow$ $\operatorname{Out}\left(N_{\mathrm{F}_{4}}(T)\right)$ [23, 20].

Proposition 5.1. There is a homotopy commutative diagram

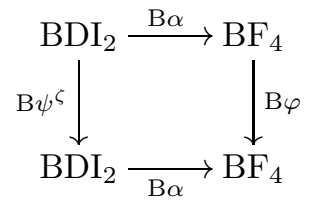

where $\zeta^{2}=-2$ and $\mathrm{B} \psi^{\zeta} \in\left[\mathrm{BDI}_{2}, \mathrm{BDI}_{2}\right]$ is the corresponding Adams map.

Proof. According to Theorem 3.3 it is enough to check the restriction to the maximal torus of $\mathrm{BDI}_{2}$, and in fact,

$$
\begin{aligned}
\pi_{2}(\mathrm{~B} T(\varphi)) \cdot \pi_{2}(\mathrm{~B} T(\alpha)) & =\left(\begin{array}{cccc}
1 & -1 & 0 & 0 \\
1 & 1 & 0 & 0 \\
0 & 0 & 1 & -1 \\
0 & 0 & 1 & 1
\end{array}\right) \cdot\left(\begin{array}{cc}
\zeta & -1 \\
-\zeta & -1+\zeta \\
0 & -1-\zeta \\
2 & -1
\end{array}\right)=\left(\begin{array}{cc}
2 \zeta & -\zeta \\
0 & -2+\zeta \\
-2 & -\zeta \\
2 & -2-\zeta
\end{array}\right) \\
& =\left(\begin{array}{cc}
-2 & 1 \\
0 & -\zeta-1 \\
-\zeta & 1 \\
-\zeta & -\zeta+1
\end{array}\right) \cdot \zeta \in W\left(\mathrm{~F}_{4}\right)\left(\pi_{2}(\mathrm{~B} T(\alpha)) \cdot \zeta\right) ;
\end{aligned}
$$

hence $\mathrm{B} \varphi \circ \mathrm{B} \alpha=\mathrm{B} \alpha \circ \mathrm{B} \psi^{\zeta}$.

Proposition 5.2. The automorphism of $H^{*}\left(\mathrm{BF}_{4} ; \mathbf{Q}_{3}\right) \cong P\left[x_{4}, x_{12}, x_{16}, x_{24}\right]$ induced by $\mathrm{B} \varphi$ is up to decomposables, determined by

$$
\mathrm{B} \varphi^{*}\left(x_{4}\right)=2 x_{4}, \quad \mathrm{~B} \varphi^{*}\left(x_{12}\right)=-8 x_{12}, \quad \mathrm{~B} \varphi^{*}\left(x_{16}\right)=16 x_{16}, \quad \mathrm{~B} \varphi^{*}\left(x_{24}\right)=-64 x_{24} .
$$

Proof. This is a long but straightforward calculation, easier done with the aid of a computer. This result coincides also with calculation done by Adams and Mahmud [1, Table 2.14]. 
Define $\mathrm{B} \tau: \mathrm{BF}_{4} \rightarrow \mathrm{BF}_{4}$ to be the automorphism $\mathrm{B} \tau=\mathrm{B} \psi^{1 / \zeta} \circ \mathrm{B} \varphi$. Then $(\mathrm{B} \tau)^{2}$ is homotopic to the identity and $\mathrm{B} \tau \circ \mathrm{B} \alpha=\mathrm{B} \alpha$ by (5.1).

Corollary 5.3. $H^{4}\left(\mathrm{~B} \tau ; \mathbf{Z}_{3}\right)$ is multiplication by -1 on $H^{4}\left(\mathrm{BF}_{4} ; \mathbf{Z}_{3}\right)$.

Proof. The effect of $H^{4}\left(\mathrm{~B} \tau ; \mathbf{Q}_{3}\right)$ on $H^{4}\left(\mathrm{BF}_{4} ; \mathbf{Q}_{3}\right)$ is given by

$$
H^{4}\left(\mathrm{~B} \tau ; \mathbf{Q}_{3}\right)=H^{4}\left(\mathrm{~B} \varphi ; \mathbf{Q}_{3}\right) \circ H^{4}\left(\mathrm{~B} \psi^{1 / \zeta} ; \mathbf{Q}_{3}\right)=2 \cdot \zeta^{-2}=-1
$$

by (5.2) and because the unstable Adams operation $\psi^{\lambda}$ induces multiplication by $\lambda^{i}$ in degree $2 i$.

Let $\tau: \mathrm{F}_{4} / \mathrm{DI}_{2} \rightarrow \mathrm{F}_{4} / \mathrm{DI}_{2}$ denote the self-homotopy equivalence of the exotic homogeneous space $\mathrm{F}_{4} / \mathrm{DI}_{2}$ induced by $\mathrm{B} \tau$ on $\mathrm{BF}_{4}$ and the identity on $\mathrm{BDI}_{2}$. This map makes the diagram

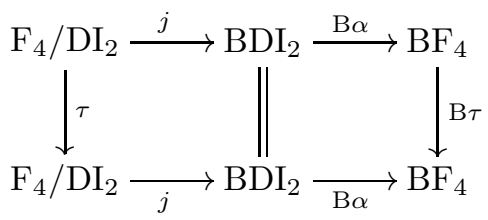

commute up to homotopy.

Corollary 5.4. The involution $H^{*}\left(\tau ; \mathbf{F}_{3}\right)$ of $H^{*}\left(\mathrm{~F}_{4} / \mathrm{DI}_{2} ; \mathbf{F}_{3}\right)$ sends the generators $z_{3}, z_{7}$, and $z_{8}$ to $-z_{3},-z_{7}$, and $-z_{8}$, respectively.

Proof. That $H^{*}\left(\tau ; \mathbf{F}_{3}\right)\left(z_{3}\right)=-z_{3}$ follows from (5.3) since (4.12) the mod 3 cohomology of $\mathrm{F}_{4} / \mathrm{DI}_{2}$ embeds in the mod 3 cohomology of $\mathrm{F}_{4}$. As the two other generators are linked to $z_{3}$ by Steenrod operations (4.11), $H^{*}\left(\tau ; \mathbf{F}_{3}\right)\left(z_{3}\right)$ must also act as multiplication by -1 on them.

We shall return to the homotopy involution $\mathrm{B} \tau$ in Section 6

\section{6. $\mathrm{BDI}_{2}$ AS A HOMOTOPY FIXED POINT SPACE}

The automorphism $\mathrm{B} \tau=\mathrm{B} \psi^{1 / \zeta_{\circ}} \mathrm{B} \phi$ of $\mathrm{BF}_{4}$ is a homotopy involution, $(\mathrm{B} \tau)^{2} \simeq 1$, that homotopy fixes $\mathrm{BDI}_{2}$ in the sense that the diagram

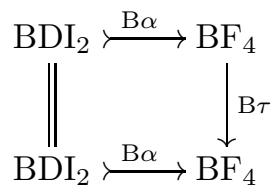

commutes up to homotopy (5.1). This suggests that $\mathrm{BDI}_{2}$ should be fixed under $\mathrm{B} \tau$ in some sense. To make this precise, we need to rigidify the above two properties.

Proposition 6.1. We may assume that the maps $\mathrm{B} \alpha$ and $\mathrm{B} \tau$ satisfy the identities $\mathrm{B} \tau \circ \mathrm{B} \alpha=\mathrm{B} \alpha$ and $\mathrm{B} \tau \circ \mathrm{B} \tau=1$.

Proof. Let $\mathbf{D}$ be the category

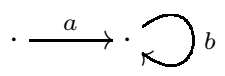

with two objects and two non-identity morphisms, $a$ and $b$, subject to the relations $b a=a$ and $b b=1$. The $\mathbf{D}$-diagram

$$
\mathrm{BDI}_{2} \stackrel{\mathrm{B} \alpha}{\longrightarrow} \mathrm{BF}_{4} \bigcirc \mathrm{B} \tau
$$


in the homotopy category of spaces is centric; in fact, all the relevant mapping spaces are weakly contractible (3.4)2) [23, 14]. Hence (6.2) has an essentially unique realization in the category of spaces [12, 1.1].

With such specific realizations of $\mathrm{B} \alpha$ and $\mathrm{B} \tau$, let $\mathrm{F}_{4} / \mathrm{DI}_{2}$ denote the homotopy fibre of $\mathrm{B} \alpha$ over any point fixed by $\mathrm{B} \tau$, e.g. any point in the image of $\mathrm{B} \alpha$. Then $\mathrm{F}_{4} / \mathrm{DI}_{2}$ is $\mathbf{Z} / 2$-space and there is a homotopy fibre sequence

$$
\mathrm{F}_{4} / \mathrm{DI}_{2} \stackrel{j}{\rightarrow} \mathrm{B} D I_{2} \stackrel{\mathrm{B} \alpha}{\longrightarrow} \mathrm{BF}_{4}
$$

of $\mathbf{Z} / 2$-spaces and $\mathbf{Z} / 2$-maps which induces [8, XI.7.1] a homotopy fibre sequence

$$
\left(\mathrm{F}_{4} / \mathrm{DI}_{2}\right)^{h \mathbf{Z} / 2} \stackrel{j^{h \mathbf{Z} / 2}}{\longrightarrow} \mathrm{BDI}_{2}^{h \mathbf{Z} / 2} \stackrel{(\mathrm{B} \alpha)^{h \mathbf{Z} / 2}}{\longrightarrow} \mathrm{BF}_{4}{ }^{h \mathbf{Z} / 2}
$$

of homotopy fixed point spaces. Here,

$$
\mathrm{BDI}_{2}^{h \mathbf{Z} / 2} \simeq \operatorname{map}\left(\mathrm{BZ} / 2, \mathrm{BDI}_{2}\right) \simeq \mathrm{BDI}_{2}
$$

since $\mathbf{Z} / 2$ acts trivially on the 3 -complete space $\mathrm{BDI}_{2}$. As to the base space, the Bousfield-Kan spectral sequence for the homotopy fixed point space $\mathrm{BF}_{4}{ }^{h \mathbf{Z} / 2}$ degenerates to the formula $\pi_{*}\left(\mathrm{BF}_{4}{ }^{h \mathbf{Z} / 2}\right)=\pi_{*}\left(\mathrm{BF}_{4}\right)^{\mathbf{Z} / 2}$. In particular, this space is non-empty and connected and its homotopy consists of the invariant part of the homotopy for $\mathrm{BF}_{4}$, cf. 38. The aim is to show that the fibre of (6.3) is contractible in order to obtain a homotopy equivalence between $\mathrm{BDI}_{2}$ and $\mathrm{BF}_{4}{ }^{h \mathbf{Z} / 2}$.

Proposition 6.2. The fibre, $\left(\mathrm{F}_{4} / \mathrm{DI}_{2}\right)^{h \mathbf{Z} / 2}$, of the fibration sequence 6.3) is contractible.

Proof. By the general result at the end of this section (6.3), it suffices to show that the space $E$ sitting in the homotopy pull back diagram

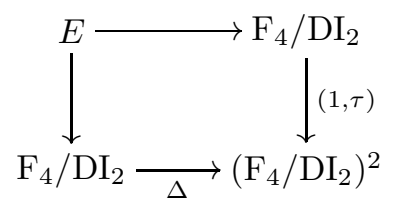

is contractible. The Eilenberg-Moore spectral sequence $E_{r}^{* *} \Rightarrow H^{*}\left(E ; \mathbf{F}_{3}\right)$ associated to (6.4) is a second quadrant cohomological spectral sequence with

$$
E_{2}^{-p q}=\operatorname{Tor}_{p}^{R \otimes R}(R, R)^{q}
$$

where $R=H^{*}\left(\mathrm{~F}_{4} / \mathrm{DI}_{2} ; \mathbf{F}_{3}\right) \cong E\left[z_{3}, z_{7}\right] \otimes P\left[z_{8}\right] /\left(z_{8}^{3}\right)$ and where $R$ is a right $R \otimes R$ module through the cup product $\mu: R \otimes R \rightarrow R$ and a left $R \otimes R$-module through $\mu \circ\left(1 \otimes \tau^{*}\right)$. We shall show that $E_{2}^{* *}=\mathbf{F}_{3}$.

Let $\kappa$ be the algebra isomorphism of $R \otimes R$ given by

$$
\kappa\left(z_{i} \otimes 1\right)=z_{i} \otimes 1-1 \otimes z_{i}, \quad \kappa\left(1 \otimes z_{i}\right)=1 \otimes z_{i}, \quad i=3,7,8 .
$$

Then $\kappa$ satisfies the identity $\varepsilon \otimes 1=\mu \kappa$, where $\varepsilon: R \rightarrow \mathbf{F}_{3}$ is the augmentation homomorphism, and hence 6.5

$$
E_{2}^{-p *}=\operatorname{Tor}_{p}^{R}\left(\mathbf{F}_{3}, R\right)
$$

where $R$ is a left $R$-module via the algebra morphism

$$
R \stackrel{1 \otimes \eta}{\longrightarrow} R \otimes R \stackrel{\kappa}{\longrightarrow} R \otimes R \stackrel{1 \otimes \tau^{*}}{\longrightarrow} R \otimes R \stackrel{\mu}{\longrightarrow} R
$$


which takes $z_{i}$ to $-z_{i}, i=3,7,8$. In particular, $R$ is a free $R$-module and the vanishing of the $E_{2}$-page for the Eilenberg-Moore spectral sequence follows.

Proof of Theorem 1.2, By (6.3, 6.2), the monomorphism $\mathrm{B} \alpha: \mathrm{BDI}_{2} \rightarrow \mathrm{BF}_{4}$ induces a homotopy equivalence $\mathrm{BDI}_{2} \simeq\left(\mathrm{BF}_{4}\right)^{h \mathbf{Z} / 2}$.

Proof of (1.2) and Corollary 1.3. Since homotopy fixed points might be seen as a homotopy limit, it is a general rule that $\operatorname{map}\left(X, Y^{h G}\right)=\operatorname{map}(X, Y)^{h G}$ and also using the Bousfield-Kan spectral sequence for the homotopy groups of a homotopy limit 8, that $\pi_{i}\left(M^{h G}\right)=\pi_{i}(M)^{G}$ when $M$ is $p$-complete and simply connected, $M^{h G}$ is not empty, and $p \nmid|G|$.

Proof of Corollary 1.4 We will prove that the map $j: \mathrm{F}_{4} / \mathrm{DI}_{2} \rightarrow \mathrm{BDI}_{2}$ is null-homotopic. For this aim we observe that both $j$ and the constant map $c$ represent classes in $\pi_{0} \operatorname{map}\left(\mathrm{F}_{4} / \mathrm{DI}_{2}, \mathrm{BDI}_{2}\right) \cong \pi_{0} \operatorname{map}\left(\mathrm{F}_{4} / \mathrm{DI}_{2}, \mathrm{BF}_{4}{ }^{h \mathbf{Z} / 2}\right)$ that map trivially down to $\pi_{0} \operatorname{map}\left(\mathrm{F}_{4} / \mathrm{DI}_{2}, \mathrm{~F}_{4}\right)$. Thus we only need to check that the map

$$
\pi_{0}\left(\operatorname{map}\left(\mathrm{F}_{4} / \mathrm{DI}_{2}, \mathrm{BF}_{4}\right)_{c}^{h \mathbf{Z} / 2}\right) \longrightarrow\left(\pi_{0} \operatorname{map}\left(\mathrm{F}_{4} / \mathrm{DI}_{2}, \mathrm{BF}_{4}\right)_{c}\right)^{\mathbf{Z} / 2}
$$

is injective. The obstructions to injectivity are given by the Bousfield-Kan spectral sequence [ㅈ, XI, $\S 7]: H^{i}\left(\mathbf{Z} / 2, \pi_{i}\left(\operatorname{map}\left(\mathrm{F}_{4} / \mathrm{DI}_{2}, \mathrm{BF}_{4}\right)_{c}\right)\right)$. The groups

$$
\pi_{i}=\pi_{i}\left(\operatorname{map}\left(\mathrm{F}_{4} / \mathrm{DI}_{2}, \mathrm{BF}_{4}\right)_{c}\right)
$$

are 3-local and nilpotent. In fact, applying $\operatorname{map}\left(\mathrm{F}_{4} / \mathrm{DI}_{2},-\right)$ to the Postnikov tower of $\mathrm{BF}_{4}$, we get a spectral sequence with $E_{2}$-page $E_{2}^{i, j} \cong H^{i}\left(\mathrm{~F}_{4} / \mathrm{DI}_{2} ; \pi_{j}\left(\mathrm{BF}_{4}\right)\right)$ converging to the homotopy of $\operatorname{map}\left(\mathrm{F}_{4} / \mathrm{DI}_{2}, \mathrm{BF}_{4}\right)_{c}$. Since the cohomology of $\mathrm{F}_{4} / \mathrm{DI}_{2}$ vanishes above certain dimension, this gives a finite filtration on $\pi_{1}$ with filtration quotients that are finitely generated modules over the 3 -adic integers.

It follows that the groups $H^{i}\left(\mathbf{Z} / 2, \pi_{i}\right)$ are trivial. Notice that $H^{1}\left(\mathbf{Z} / 2, \pi_{1}\right)$ might be a non-abelian first cohomology group. For this we refer to [27, §5, Proposition $38]$, and use induction over the nilpotence class of $\pi_{1}$.

The splitting is now constructed using a section of $\pi: \mathrm{F}_{4} \rightarrow \mathrm{F}_{4} / \mathrm{DI}_{2}$ and the $H$-space structure of $\mathrm{F}_{4}$.

In the homotopy splitting from Corollary 1.4 of $\mathrm{F}_{4}, \mathrm{~F}_{4} / \mathrm{DI}_{2} \simeq K(3)$ is one of Harper's $H$-spaces (7.5) and $\mathrm{DI}_{2} \simeq B_{5}(3)$ is one of the Mimura-Toda bundles of completed spheres over spheres [22].

We finish this section with the general results that we used above to compute the homotopy fixed point space $\left(\mathrm{F}_{4} / \mathrm{DI}_{2}\right)^{h \mathbf{Z} / 2}$.

First, let $G$ be a finite group and $K$ a $G$-space. Define $E$ to be the homotopy pullback

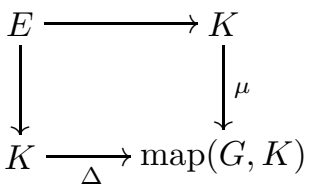

where $\Delta$ takes $x \in K$ to the constant map with value $x$ and the action map $\mu$ takes $x$ to the map $\mu(k)(h)=h^{-1} x, h \in G$. These two maps are equivariant if we equip $\operatorname{map}(G, K)$ with the action $(g u)(h)=u\left(g^{-1} h\right), g, h \in G, u \in \operatorname{map}(G, K)$, and $K$ in the lower left corner with the trivial action. Thus (6.5) is a diagram of $G$-spaces and $G$-maps. 
Theorem 6.3. If $K$ is $\mathrm{B} G$-null, the $G$-map $E \rightarrow K$ induces a weak homotopy equivalence $E^{h G} \rightarrow K^{h G}$ of homotopy fixed point spaces.

Proof. Since the homotopy fixed point functor commutes with homotopy pull-backs 8, XI.4.3], there is an induced homotopy pull-back diagram

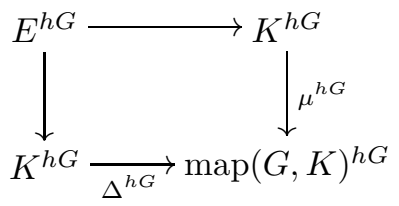

of homotopy fixed point spaces. The map $\Delta^{h G}: K^{h G} \rightarrow \operatorname{map}(G, K)^{h G}$ can be identified to the evaluation map $\operatorname{map}(\mathrm{B} G, K) \rightarrow K$. Indeed, $K^{h G}=\operatorname{map}(\mathrm{B} G, K)$, since the action is trivial in the lower left corner, and $\operatorname{map}(G, K)^{h G}=\operatorname{map}\left(G_{h G}, K\right)=$ $\operatorname{map}(*, K)=K$. If $K$ is $\mathrm{B} G$-null, $\Delta^{h G}$ is a homotopy equivalence and so is then the top horizontal map $E^{h G} \rightarrow K^{h G}$.

By an argument dual to that of [21, 5.1] [28, p. 282], we may identify $E$ and the homotopy limit of the diagram $K \stackrel{G}{\rightarrow} K$ consisting of the $|G|$ maps $K \rightarrow K$ given by $x \rightarrow g x$ for $g \in G$.

The final result that we used above was an algebraic computation ensuring the vanishing of an Eilenberg-Moore spectral sequence.

Let $R$ be a unital and augmented (graded) algebra over a field $k$. The structure maps

$$
\mu: R \otimes R \rightarrow R, \quad \eta: k \rightarrow R, \quad \varepsilon: R \rightarrow k
$$

make $R$ a right $R \otimes R$-module and $k$ a right $R$-module.

For any right $R$-module $A$, the tensor product $A \otimes R$ is a right $R \otimes R$-module with right $R \otimes R$-multiplication given by

$$
(a \otimes r) \cdot(s \otimes t)=a s \otimes r t
$$

for all elements $a \in A, r, s, t \in R$ (modified by the usual sign in the graded case). When $A=k$, in particular, right multiplication in $k \otimes R=R$ by $s \otimes t \in R \otimes R$ is right multiplication in $R$ by $(\varepsilon \otimes 1)(s \otimes t)=\varepsilon(s) t$.

Lemma 6.4. Let $\kappa$ be an algebra isomorphism of $R \otimes R$ and $\otimes_{R}(R \otimes R)$ the left adjoint functor to the forgetful functor induced by the algebra homomorphism $\kappa \circ(1 \otimes \eta): R \rightarrow R \otimes R$. Then

1. $A \otimes_{R}(R \otimes R)=A \otimes R$ with right $R \otimes R$-multiplication $(a \otimes r)(s \otimes t)=$ $(a \otimes r) \cdot \kappa^{-1}(s \otimes t)$

2. $\operatorname{Tor}_{p}^{R}(A, B)=\operatorname{Tor}_{p}^{R \otimes R}\left(A \otimes_{R}(R \otimes R), B\right)$ and

$$
\operatorname{Ext}_{R}^{p}(A, B)=\operatorname{Ext}_{R \otimes R}^{p}\left(A \otimes_{R}(R \otimes R), B\right)
$$

for all right $R$-modules $A$, all left $R \otimes R$-modules $B$, and all $p \geq 0$.

Proof. We have

$$
U(\kappa(1 \otimes \eta))=U(1 \otimes \eta) U(\kappa) \quad \text { and } \quad U(\kappa) L(\kappa(1 \otimes \eta))=L(1 \otimes \eta)
$$

where $L(f)$ denotes the left adjoint to the forgetful functor $U(f)$ induced by the algebra homomorphism $f$. Note that $L(1 \otimes \eta)(A)=A \otimes R$. It follows that $L(1 \otimes \eta)$ is exact; it also takes $R$-projectives to $R \otimes R$-projectives because it is left adjoint to a (right) exact functor [33, 2.3.10]. The same is true of the functor $L(\kappa(1 \otimes \eta))$ 
which differs from $L(1 \otimes \eta)$ by an isomorphism. Similarly, $U(\kappa(1 \otimes \eta))$ is an exact functor that takes injectives to injectives. Therefore, the identities

$$
A \otimes_{R} U B=L A \otimes_{R \otimes R} B, \quad \operatorname{Hom}_{R}(A, U B)=\operatorname{Hom}_{R \otimes R}(L A, B)
$$

where $L=L(\kappa(1 \otimes \eta))$ and $U=U(\kappa(1 \otimes \eta))$, prolong to the identities of (6.412).

Corollary 6.5. Suppose in addition to the assumptions of (6.4) that $\varepsilon \otimes 1=\mu \kappa$. Then $\kappa \circ(1 \otimes \eta)$ induces an isomorphism

$$
\operatorname{Tor}_{p}^{R}(k, B)=\operatorname{Tor}_{p}^{R \otimes R}(R, B)
$$

for all left $R \otimes R$-modules $B$ and all $p \geq 0$.

Proof. Note that $L(1 \otimes \eta)(k)=U(\varepsilon \otimes 1)(R)$ where $R$ is considered a module over itself. Therefore $L(\kappa(1 \otimes \eta))(k)=U\left(\kappa^{-1}\right) L(1 \otimes \eta)(k)=U\left(\kappa^{-1}\right) U(\varepsilon \otimes 1)(R)=$ $U\left((\varepsilon \otimes 1) \kappa^{-1}\right)(R)=U(\mu)(R)$ which is $R$ as an $R \otimes R$-module.

\section{HARPER'S MOlecule}

This section contains some comments on John Harper's work on finite $H$-spaces. Our starting point will be the theorem below and our main result (7.3) says that the space $K(p)$ is cohomologically unique in that it is determined up to homotopy equivalence by cohomological information. This will enable us (7.5) to identify $K(3)$ and the fibre $\mathrm{F}_{4} / \mathrm{DI}_{2}$ of the embedding $\mathrm{B} \alpha: \mathrm{BDI}_{2} \rightarrow \mathrm{BF}_{4}$.

Theorem 7.1 ([17, Theorem B]). For each odd prime $p$ there exists a simply connected finite complex $K(p)$ whose p-localization is an $H$-space and with

$$
\begin{aligned}
H^{*}\left(K(p) ; \mathbf{F}_{p}\right) & \cong E\left[x_{3}, x_{2 p+1}\right] \otimes P\left[x_{2 p+2}\right] /\left(x_{2 p+2}\right)^{p}, \\
P^{1} x_{3} & =x_{2 p+1}, \beta x_{2 p+1}=x_{2 p+2} .
\end{aligned}
$$

It turns out that the $p$-completed homotopy type of the space $K(p)$ is determined by its $\bmod p$ cohomology as an algebra over the Steenrod algebra. This is proved by means of classical homotopy theory methods and we are sure that it is known to J. Harper and perhaps other people. We will provide a proof for the sake of completeness.

For this aim we sketch the construction of this $p$-completed homotopy type by G.E. Cooke and L. Smith [10]. They first introduce the stable two-stage Postnikov system

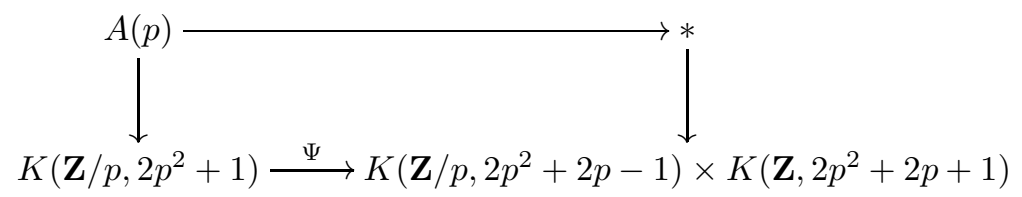

where $\Psi=\left(P^{1} \iota, \beta P^{1} \beta \iota\right)$ (Notice that $\beta P^{1} \beta \iota \in H^{2 p^{2}+2 p+1}\left(\mathbf{Z} / p, 2 p^{2}+1 ; \mathbf{Z} / p\right)$ is a $\bmod p$ reduction of an integral class.) This is constructed in such a way that the composition

$$
K(\mathbf{Z}, 3) \stackrel{P^{p} P^{1} \iota}{\longrightarrow} K\left(\mathbf{Z} / p, 2 p^{2}+1\right) \stackrel{\Psi}{\longrightarrow} K\left(\mathbf{Z} / p, 2 p^{2}+2 p-1\right) \times K\left(\mathbf{Z}, 2 p^{2}+2 p+1\right)
$$


is null-homotopic and therefore the first map lifts (non-uniquely) to $\varphi_{p}$ :

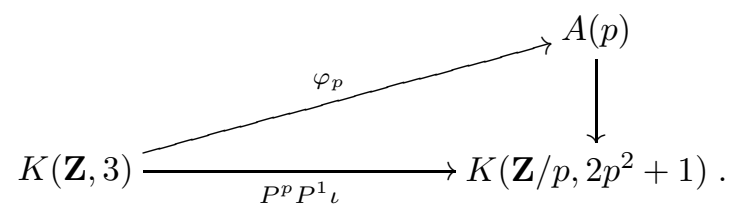

Now define $Y(p)$ as the homotopy fibre

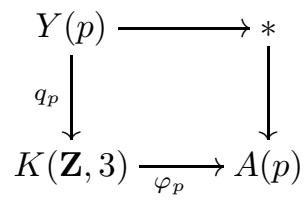

of the map $\varphi_{p}$.

The cohomology of the two-stage Postnikov system $A(p)$ is computed from results of [30]:

$$
H^{*}\left(A(p) ; \mathbf{F}_{p}\right) \cong E[\iota] \otimes P\left[\beta \iota, P^{1} \beta \iota\right] \otimes H
$$

where $\iota$ is restricted from the fundamental class of $K\left(\mathbf{Z} / p, 2 p^{2}+1\right)$ and, in particular, $\operatorname{deg} \iota=2 p^{2}+1$ and $H$ is a Hopf algebra that is $\left(2 p^{2}+4 p-2\right)$-connected. Now, $\varphi_{p}^{*}(\iota)=P^{p} P^{1} \iota_{3}, \varphi_{p}^{*}(\beta \iota)=\beta P^{p} P^{1} \iota_{3}$ and $\varphi_{p}^{*}\left(P^{1} \beta \iota\right)=P^{1} \beta P^{p} P^{1} \iota_{3}=\left(\beta P^{1} \iota_{3}\right)^{p}$, from which it is computed the cohomology of $Y(p)$ in low dimensions:

Proposition $7.2([10,1.2])$. In dimensions less than or equal to $2 p^{2}+2 p+2$ the $\bmod p$ cohomology of $Y(p)$ coincides with $E\left[u, P^{1} u\right] \otimes P\left[\beta P^{1} u\right] /\left(\beta P^{1} u\right)^{p}$ where $u=q_{p}^{*} \iota_{3}$.

It then follows that stage $2 p^{2}+2 p+2$ in the homology decomposition tower [18, Chap. 8] [5] for $Y(p)$ is a space $K(p)=Y(p)^{2 p^{2}+2 p+2}$ with

$$
H^{*}\left(K(p) ; \mathbf{F}_{p}\right) \cong E\left[u, P^{1} u\right] \otimes P\left[\beta P^{1} u\right] /\left(\beta P^{1} u\right)^{p} .
$$

Dually, the Postnikov tower for $K(p)$ has the form

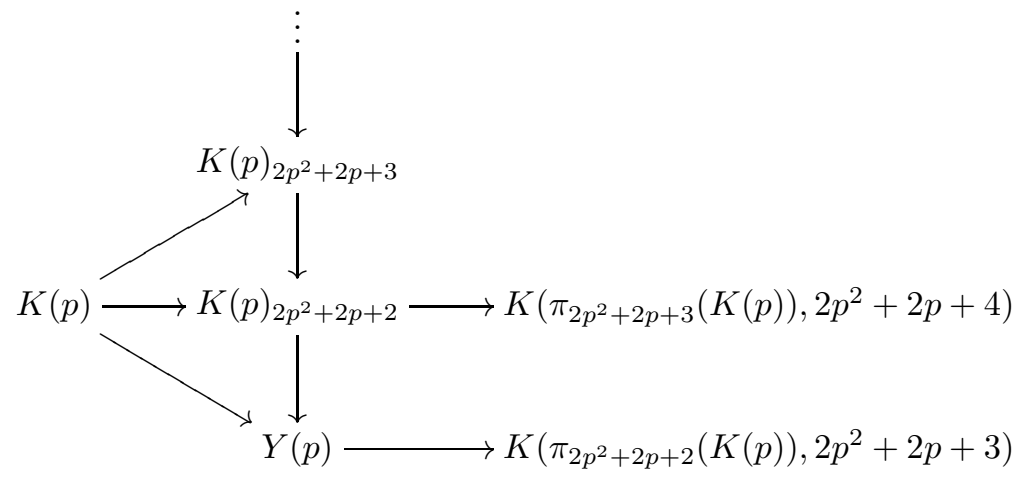

because $K(p)_{2 p^{2}+2 p+1}=Y(p)_{2 p^{2}+2 p+1}^{2 p^{2}+2 p+2}=Y(p)_{2 p^{2}+2 p+1}=Y(p)$ appears at stage $2 p^{2}+2 p+1$.

We now come to our main result of this section which is homotopy uniqueness at the prime $p$ for $K(p)_{p}^{\wedge}$. 
Theorem 7.3. Let $X$ be a p-complete space with $H^{*}\left(X ; \mathbf{F}_{p}\right) \cong H^{*}\left(K(p) ; \mathbf{F}_{p}\right)$ as algebras over the Steenrod algebra. Then $X \simeq K(p)_{p}^{\wedge}$.

Proof. Assume that $X$ is a $p$-complete space with

$$
H^{*}\left(X ; \mathbf{F}_{p}\right) \cong E\left[x_{3}, x_{2 p+1}\right] \otimes P\left[x_{2 p+2}\right] /\left(x_{2 p+2}\right)^{p}
$$

satisfying $P^{1} x_{3}=x_{2 p+1}$ and $\beta x_{2 p+1}=x_{2 p+2}$. Notice that $X$ is 2-connected and since the mod $p$ cohomology is finite dimensional, each $\pi_{i}(X)$ and $H^{i}\left(X ; \mathbf{Z}_{p}\right)$ is a finitely generated $\mathbf{Z}_{p}$-module.

The Bockstein spectral sequence with $p$-adic coefficients applies. It collapses at

$$
B_{\infty} \cong B_{2} \cong E\left[x_{3}, x_{2 p+1}\left(x_{2 p+2}\right)^{p-1}\right]
$$

and shows that the top integral class is $H_{2 p^{2}+2 p+2}\left(X ; \mathbf{Z}_{p}\right) \cong H^{2 p^{2}+2 p+2}\left(X ; \mathbf{Z}_{p}\right) \cong$ $\mathbf{Z}_{p}$. (The cohomological dimension $\operatorname{cd}_{\mathbf{z}_{p}}(X)=2 p^{2}+2 p+2$.) In particular, $H^{>2 p^{2}+2 p+2}(X ; M)=0$ for any $\mathbf{Z}_{p}$-module $M$ and thus $\left[X, K(p)_{p}^{\wedge}\right]=\left[X, Y(p)_{p}^{\wedge}\right]$ by obstruction theory.

We now use the $p$-completed version of the Cooke-Smith construction of $K(p)$. Let

$$
X \stackrel{x_{3}}{\longrightarrow} K\left(\mathbf{Z}_{p}, 3\right)
$$

represent a generator of $H^{3}\left(X ; \mathbf{Z}_{p}\right) \cong \mathbf{Z}_{p}$.

Claim 7.4. $\left[X, A(p)_{p}^{\wedge}\right]=\{*\}$.

Proof. This follows from the exact sequence of sets

$$
0=H^{2 p^{2}+2 p-2}\left(X ; \mathbf{F}_{p}\right) \times H^{2 p^{2}+2 p}\left(X ; \mathbf{F}_{p}\right) \rightarrow\left[X, A(p)_{p}^{\wedge}\right] \rightarrow H^{2 p^{2}+1}\left(X ; \mathbf{F}_{p}\right)=0
$$

obtained by mapping $X$ into the fibration $K\left(\mathbf{Z} / p, 2 p^{2}+2 p-2\right) \times K\left(\mathbf{Z}_{p}, 2 p^{2}+2 p\right) \rightarrow$ $A(p)_{p}^{\wedge} \rightarrow K\left(\mathbf{Z} / p, 2 p^{2}+1\right)$.

Since $\varphi_{p} x_{3}$ is null-homotopic by Claim $7.4 x_{3}: X \rightarrow K\left(\mathbf{Z}_{p}, 3\right)$ lifts to a map

$$
f: X \rightarrow Y(p)_{p}^{\wedge}
$$

that satisfies $f^{*}(u)=x_{3}$. Hence $f^{*}$ is an isomorphisms in dimensions $\leq 2 p^{2}+2 p+2$ and the corresponding map $X \rightarrow K(p)_{p}^{\wedge}$ an isomorphism on mod $p$ cohomology.

In the special case where $p=3$, we note that Harper's molecule $K(3) \wedge$ and the exotic homogeneous space $\mathrm{F}_{4} / \mathrm{DI}_{2}$ have isomorphic mod 3 cohomology algebras over the Steenrod algebra. Thus we may conclude that these two spaces are homotopy equivalent.

Proposition 7.5. $\mathrm{F}_{4} / \mathrm{DI}_{2}$ and $K(3)_{3}^{\wedge}$ are homotopy equivalent 3 -complete spaces.

We do not know if $K(p)$ for $p>3$ is an exotic homogeneous space with respect to some monomorphism of $p$-compact groups.

\section{REFERENCES}

1. J.F. Adams and Z. Mahmud, Maps between classifying spaces, Invent. Math. 35 (1976), 1-41. MR 54:11331

2. J. F. Adams and C. W. Wilkerson, Finite H-spaces and algebras over the Steenrod algebra, Ann. of Math. (2), 111 (1980), 95-143. MR 81h:55006

3. J. Aguadé, Constructing modular classifying spaces, Israel J. Math. 66 (1989), 23-40. MR 90m:55016 
4. K.K.S. Andersen, The normalizer splitting conjecture for p-compact groups, Fund. Math. 161 (1999), 1-16. MR 2000:02

5. Marc Aubry, Homotopy theory and models, Birkhäuser Verlag, Basel, 1995, Based on lectures held at a DMV seminar in Blaubeuren by H. J. Baues, S. Halperin and J.-M. Lemaire. MR 96g:55001

6. A. Borel, Sur la cohomologie des espaces fibrés principaux et des espaces homogènes de groupes de Lie compacts, Ann. of Math. (2) 57 (1953), 115-207. MR 14:490e

7. N. Bourbaki, Groupes et algèbres de Lie, Chp. 9, Masson, Paris, 1982. MR 84i:22001

8. A.K. Bousfield and D.M. Kan, Homotopy limits, completions and localizations, 2nd ed., Lecture Notes in Mathematics, vol. 304, Springer-Verlag, Berlin-Heidelberg-New York-LondonParis-Tokyo, 1987. (1st ed.) 1972. MR 51:1825

9. A. Clark and J.R. Ewing, The realization of polynomial algebras as cohomology rings, Pacific J. Math 50 (1974), 425-434. MR 51:4221

10. George E. Cooke and Larry Smith, On realizing modules over the Steenrod algebra, J. Pure Appl. Algebra 13 (1978), no. 1, 71-100. MR 80h:55018

11. L.E. Dickson, A fundamental system of invariants of the general modular linear group with a solution of the form problem, Trans. Amer. Math. Soc. 12 (1911), 75-98.

12. W.G. Dwyer and D.M. Kan, Centric maps and realizations of diagrams in the homotopy category, Proc. Amer. Math. Soc. 114 (1992), 575-584. MR 92e:55011

13. W.G. Dwyer and C.W. Wilkerson, Homotopy fixed point methods for Lie groups and finite loop spaces, Ann. of Math. (2) 139 (1994), 395-442. MR 95e:55019

14. - The center of a p-compact group, The Čech Centennial. Contemporary Mathematics, vol. 181 (Providence, Rhode Island) (M. Cenkl and H. Miller, eds.), American Mathematical Society, 1995, pp. 119-157. MR 96a:55024

15. Eric M. Friedlander, Exceptional isogenies and the classifying spaces of simple Lie groups, Ann. Math. (2) 101 (1975), 510-520. MR 52:11900

16. John R. Harper, The mod 3 homotopy type of $F_{4}$, Lecture Notes in Math., Vol. 418 (1974), 58-67. MR 51:1810

17. $\longrightarrow$ - H-spaces with torsion, Mem. Amer. Math. Soc. 22 (1979), no. 223, viii+72. MR 80k:55033

18. Peter Hilton, Homotopy theory and duality, Gordon and Breach Science Publishers, New York, 1965. MR 33:6624

19. Stefan Jackowski, James McClure, and Bob Oliver, Maps between classifying spaces revisited, The Čech centennial (Boston, MA, 1993), Contemp. Math., vol. 181, Amer. Math. Soc., Providence, RI, 1995, pp. 263-298. MR 96a:55027

20. Stefan Jackowski, James McClure, and Bob Oliver, Self homotopy equivalences of classifying spaces of compact connected Lie groups, Fund. Math. 147 (1995), 99-126. MR 96f:55009

21. Stefan Jackowski and James E. McClure, Homotopy approximations for classifying spaces of compact Lie groups, Algebraic topology (Arcata, CA, 1986), Springer, Berlin, 1989, pp. 221-234. MR 90m:55012

22. Mamoru Mimura and Hirosi Toda, Cohomology operations and homotopy of compact Lie groups. I, Topology 9 (1970), 317-336. MR 42:1144

23. J.M. Møller, $N$-determined $p$-compact groups, to appear in Fund. Math.

24. Rational isomorphisms of p-compact groups, Topology 35 (1996), 201-225. MR 97b:55019

25. Toric morphisms of p-compact groups, Preprint, November 1997, to appear in the Proceedings of the 1998 Barcelona Conference on Algebraic Topology.

26. J.M. Møller and D. Notbohm, Centers and finite coverings of finite loop spaces, J. Reine Angew. Math. 456 (1994), 99-133. MR 95j:55029

27. Jean-Pierre Serre, Cohomologie Galoisienne, Lecture Notes in Mathematics, vol. 5, SpringerVerlag, Berlin-Heidelberg-New York-Tokyo, 1973. MR 53:8030

28. Jolanta Słomińska, Homotopy colimits on E-I-categories, Algebraic topology Poznań 1989, Lecture Notes in Math., vol. 1474, Springer, Berlin, 1991, pp. 273-294. MR 92g:55023

29. L. Smith and R.M. Switzer, Realizability and nonrealizability of Dickson algebras, Proc. Amer. Math. Soc. 89 (1983), 303-313. MR 85e:55036

30. Larry Smith, The cohomology of stable two stage Postnikov systems, Illinois J. Math. 11 (1967), 310-329. MR 34:8406 
31. _ Polynomial invariants of finite groups, Research Notes in Mathematics, vol. 6, A K Peters Ltd., Wellesley, MA, 1995. MR 96f:13008

32. Norman Steenrod, Polynomial algebras over the algebra of cohomology operations, H-spaces (Actes Réunion Neuchâtel, 1970), Springer, Berlin, 1971, Lecture Notes in Mathematics, Vol. 196, pp. 85-99. MR 44:3316

33. Charles A. Weibel, An introduction to homological algebra, Cambridge Studies in Advanced Mathematics, vol. 38, Cambridge University Press, Cambridge, 1994. MR 95f:18001

34. Clarence Wilkerson, Some polynomial algebras over the Steenrod algebra $A_{p}$, Bull. Amer. Math. Soc. 79 (1973), 1274-1276 (1974). MR 49:3938

35. - A primer on the Dickson invariants, Proceedings of the Northwestern Homotopy Theory Conference (Evanston, Ill., 1982) (Providence, R.I.), Amer. Math. Soc., 1983, pp. 421-434. MR 85c:55017

36. Clarence W. Wilkerson, Integral closure of unstable Steenrod algebra actions, J. Pure Appl. Algebra 13 (1978), no. 1, 49-55. MR 58:24266

37. Zdzisław Wojtkowiak, On maps from holim $F$ to $Z$, Algebraic topology, Barcelona, 1986, Lecture Notes in Math., vol. 1298, Springer, Berlin, 1987, pp. 227-236. MR 89a:55034

38. A. Zabrodsky, On the realization of invariant subgroups of $\pi_{*}(X)$, Trans. Amer. Math. Soc. 285 (1984), no. 2, 467-496. MR 85k:55012

Departament de Matemàtiques, Universitat Autònoma de Barcelona, 08193 BellaTERRA, SPAIN

E-mail address: broto@mat.uab.es

Matematisk Institut, Universitetsparken 5, DK-2100 København $\varnothing$, Denmark

E-mail address: moller@math.ku.dk 